\title{
Calories on menus in Ireland - who's counting?
}

\author{
G.D. Ussher ${ }^{1}$, S.E. Kielthy ${ }^{1}$, K.A. Emerson ${ }^{1}$, F.E. Douglas ${ }^{1}$, O.C. Lyons ${ }^{1,2}$ and M.A.T. Flynn ${ }^{1,2}$ \\ ${ }^{1}$ Public Health Nutrition, Food Safety Authority of Ireland, Dublin 1, Republic of Ireland and ${ }^{2}$ Northern Ireland Centre \\ for Food and Health, University of Ulster, Coleraine BT52 1SA, UK
}

Calorie menu labelling (CML) can encourage people to purchase fewer calories ${ }^{(1)}$. More males than females are affected by overweight and obesity in Ireland ${ }^{(2)}$ but males tend to regard calorie information as being 'just for women'(3). This study describes the calorie content of foods purchased by males and females in a hospital staff/ visitor canteen serving breakfast and lunch, before and after the implementation of CML.

Calories per portion of food served for breakfast (fixed daily menu) and lunch (variable daily menu, three week menu cycle), were calculated using MenuCal ${ }^{\circledR(4)}$. Consumer food choices were recorded for both meals on five days (Monday-Friday) of the same menu cycle, pre-, and six weeks post-, CML implementation. The variation in calories purchased pre- and post- CML was analysed according to gender, meal, day, service counter (hot [kcals tended to be higher] $v s$. cold) and food group as defined in Ireland's healthy eating guidelines $^{(5)}$.

Data was collected for 999 customers pre-, and 1005 customers post-, CML. Immediately following implementation of CML, and before impact was evaluated, the portion of baked potato on offer was reduced by a third due to customer demand. Evaluation of customer purchasing behaviour showed a significant decrease in median calories purchased after CML was implemented (599kcal vs. $530 \mathrm{kcal}, \mathrm{p}<0.05)$. As shown in the table this decrease was more evident in males.

\begin{tabular}{|c|c|c|c|c|c|c|c|c|}
\hline & \multirow[b]{2}{*}{$(n)$} & \multicolumn{3}{|l|}{ Males } & \multirow[b]{2}{*}{$(n)$} & \multicolumn{3}{|l|}{ Females } \\
\hline & & $\begin{array}{l}\text { Pre } \mathrm{CML}^{\dagger} \\
\text { Median (Range) }\end{array}$ & $(n)$ & $\begin{array}{l}\text { Post CML } \\
\text { Median (Range) }\end{array}$ & & $\begin{array}{l}\text { Pre CML } \\
\text { Median (Range) }\end{array}$ & $(n)$ & $\begin{array}{l}\text { Post CML } \\
\text { Median (Range) }\end{array}$ \\
\hline Breakfast (kcal) & 214 & $598(29-2700)$ & 180 & $585(2-2112)$ & 318 & $419(2-5050)$ & 238 & $406(2-2018)$ \\
\hline Lunch (kcal) & 172 & $813(16-2079)$ & 229 & $622(4-2840)^{* * *}$ & 295 & $635(15-1612)$ & 358 & $551(10-2109)^{* *}$ \\
\hline Both meals (kcal) & 386 & $668(16-2700)$ & 409 & $612(2-2840)^{*}$ & 613 & $530(2-5050)$ & 596 & $496(2-2109)$ \\
\hline
\end{tabular}

† Calorie Menu Labelling

Median values were statistically different (Mann-Whitney U) ${ }^{*} \mathrm{p}<0.05,{ }^{* *} \mathrm{p}=0.001,{ }^{* * *} \mathrm{p}<0.001$

At lunch following CML, females purchased significantly fewer median calories on Monday (719 kcal vs. 449kcal, p < 0.001) but more on Friday $(511 \mathrm{kcal} v s .706 \mathrm{kcal}, \mathrm{p}<0.05)$. Over both meals significantly fewer males chose hot counter options after CML $(74 \%$ vs. $68 \%, \mathrm{p}<0 \cdot 01)$. At lunch, significantly fewer customers chose hot counter options $(74 \% v s .57 \%$, p < 0.001$)$ while more chose from the cold counter when calories were displayed $(25 \%$ vs. $35 \%, \mathrm{p}<0.001)$. Following CML significantly fewer customers chose foods from the 'other' food group i.e. high fat/ high sugar foods $(31 \% v s .23 \%, \mathrm{p}<0.001)$, with a specific reduction in the proportion of customers choosing chips ( $39 \%$ to $26 \%, \mathrm{p}<0.001)$. More consumers chose foods from the 'fruit and vegetable' food group (44 \% vs. $57 \%, \mathrm{p}<0.001)$ after CML.

In conclusion following CML customers made healthier choices and fewer calories were purchased, particularly by males. A 'Friday feeling' effect was observed for females.

1. Bollinger B, Leslie P, Sorenson A (2011) Amer Econ J 3, 91-128

2. National Adult Nutrition Survey. (2011). Available from: http://www.iuna.net/wp-content/uploads/2010/12/National-Adult-Nutrition-SurveySummary-Report-March-2011.pdf (Accessed March 2015)

3. Ray K, Clegg S, Davidson R et al. (2013) Evaluation of Caloriewise. Available from: http://www.food.gov.uk/northern-ireland/researchni/fs307001. (Accessed March 2015).

4. MenuCal ${ }^{\circledR}$. Available from: www.menucal.ie (Accessed Oct 2014).

5. Flynn MAT, O’Brien CM, Ross V et al. (2012) Public Health Nutr 15, 527-37. 